professionals and guideline developers; 6. Processing results and preparation of field tests: sorting, selection and reduction of instruments for domains and concepts by using the framework and criteria for feasibility and use in daily practice; 7. Implementation- and dissemination planning.

Results A Conceptual framework based on the structure of clinical reasoning and International Classification of Functioning. An inventory of domains that are relevant for physiotherapy and feasibility criteria for the selection of instruments. A guide for the selection and reduction of recommended instruments in guidelines and use in daily practice.

Conclusions The framework gives professionals and guideline developers the same structure and a clearer understanding about the selection of instruments for daily practice. It helps professionals to learn when to use which instrument for what patient.

\section{P189 ISCHEMIC STROKE: THROUGH ADAPTED CLINICAL GUIDELINE TO LOCAL CLINICAL PROTOCOLS}

A Morozov, A Stepanenko, N Chagarna, O Shylkina. The State Expert Center of the Ministry of Health of Ukraine, Kyiv, Ukraine

\section{0:1136/bmjqs-2013-002293.198}

Background The health care system of Ukraine required improvement in treatment of stroke patients based on evidence present in GIN.

Objectives To compare Ukrainian treatment practices with the best strategies for stroke management and to improve quality of health care on the basis of the identified differences.

Methods Multidisciplinary working group has prepared adapted guidelines "Modern principles of diagnosis and management of patients with acute ischemic stroke and TIA", "Recommendations for the management of patients with ischemic stroke and TIA", "Rehabilitation, prevention and management of complications and discharge planning in ischemic stroke" with regard of the evidence-based strategies for stroke treatment. Simultaneously, unified clinical protocols of medical care "Ischemic stroke" and "Systemic thrombolysis for stroke" were developed on the basis of the guidelines and then approved by the Ministry of Health of Ukraine in August 2012.

Results There were identified differences in the stroke treatment in Ukraine compared to the best practice, which resulted in amendments to the corresponding regulations in health care. In order to integrate these changes into clinical practice local protocols and critical pathways for management of stroke are developing in all healthcare facilities taking into account peculiarities of the region and available resources of the hospitals.

Discussion Local protocols comprise evidence-based statements which represent the best practice from clinical guidelines from GIN and other databases.

Implications for Guideline Developers/Users Measures enabling changes in existing medical practice are identified to ensure effective treatment of stroke patients within 4.5 hours after symptoms onset.

\section{P190 REGISTRY OF MEDICAL TECHNOLOGICAL DOCUMENTS FOR SUPPORTING GUIDELINES ACCESSIBILITY}

A Morozov, A Stepanenko, O Lishchyshyna,Y Gorokh, V Khachaturyan. The State Expert Center of the Ministry of Health of Ukraine, Kyiv, Ukraine

10:1136/bmjqs-2013-002293.199
Background The Ministry of Health of Ukraine has approved the methodology for development of medical and technological documents on the basis of evidence. The first documents on the basis of evidence were developed by multidisciplinary working groups in recent years. We can witness the process of guidelines adaptation and their implementation into health care practice.

Context It is necessary to ensure the availability of new documents for doctors, health care providers, and public. Transparency at all stages of medical and technical documents development is also very important.

Description of Best Practice The website of the registry of medical and technological documents has been created and posted at http://www.dec.gov.ua/mtd/index.html. The website includes: Adapted clinical guidelines and unified clinical protocols of medical care developed by multidisciplinary working groups; - Draft documents submitted for public discussion; - Information on clinical topics under consideration; - Methodological materials for developers - members of multidisciplinary working groups; Links to international sources of evidential information. The development of the content of the website continues.

Lessons for Guideline Developers, Adaptors, Implementers, and/ or Users Creating a specialised website that integrates methodological materials, adapted clinical guidelines, unified clinical protocols, and information about the documents under consideration provides accessibility of documents, convenience for users, and improves confidence of professionals and the public in new documents.

\section{P193 ADOPTING NICE GUIDELINES IN OTHER COUNTRIES}

M Gholitabar, H McGuire. National Collaborating Centre for Women's and Children's Health (NCC-WCH), London, UK

\section{0:1136/bmjqs-2013-002293.200}

Background Although NICE guidelines are developed to inform clinical practice in England and Wales, they attract interest from researchers, clinicians and healthcare organisations all over the world. This has generated discourse about whether, and to what degree, recommendations from NICE guidelines are applicable to different countries.

Objectives To consider if NICE recommendations should be adapted for use in other countries in terms of differences in health care systems, values and needs? - To discuss the varying approaches to adopting NICE guidelines and recommendations Methods Using the NICE Intrapartum Care clinical guideline, we will select illustrative examples of recommendations. Factual data and information from one developing country - Islamic Republic of Iran - will be used to evaluate the ease with which these could be implemented. We will consider the Iranian health care setting; economic situation; social values; geographical issues; cultural issues and priority health care policies.

Results We will present the findings in three categories corresponding to the ease with which NICE recommendations can be implemented in another country.

Discussion A focused discussion will centre on - whether adopting NICE guidelines and recommendations outside of England and Wales is feasible - what additional work may be need to carried out to facilitate this process - the ethics of such activity in terms of self-reliance and research capacity.

Implications for Guideline Developers/Users This project will identify areas where existing guidance can be shared across borders thereby reducing duplication of effort; facilitating 
international collaboration and building capacity in guideline development methodology and implementation.

\section{P196 UPDATING THE EVIDENCE FOR CPGS: THE USEFULNESS OF RSS FEEDS}

${ }^{1} \mathrm{I}$ Etxeandia-lkobaltzeta, ${ }^{1} \mathrm{~N}$ Ibargoyen-Roteta, ${ }^{2} \mathrm{R}$ Roteche-del Campo, ${ }^{3} \mathrm{~A}$ Etxeberria-Agirre, ${ }^{4} \mathrm{M}$ Callén-Blecua. 'Basque Office for Health Technology Assessment (OSTEBA)," VitoriaGasteiz, Spain; ${ }^{2}$ Primary Care Centre of Alza (Osakidetza - NHS), Alza, Spain; ${ }^{3}$ Primary Care Centre of Hernani (Osakidetza - NHS), Hernani, Spain; ${ }^{4}$ Primary Care Centre of Bidebieta (Osakidetza - NHS), Bidebieta, Spain

\section{0:1136/bmjqs-2013-002293.201}

Background Scientific knowledge is in constant evolution and even if Clinical Practice Guidelines (CPGs) get out of date in five years time, there is a lack of standardised method for an efficient updating process.

Context A proposal used to update efficiently the bibliographic searches during the development of a CPG on childhood asthma management is described.

Description of Best Practice Bibliographic searches were performed in Medline, Embase and All EBM databases using Ovid, and in BMJ update database. Search strategies and RSS-feeds were created and saved when possible. E-mail alerts for those searches carried out in databases with no RSS services were also created. All the RSS-feeds were centralised in a Netvibes web portal and organised into tabs (one tab by clinical question). New alerts were revised periodically during the guideline development process and new references incorporated in the guideline when relevant. RSS-feeds could be created for Medline and Embase searches. The centralization of RSS-feeds in a Netvibes portal facilitated the reviewing process of the latest published articles by clinical question. The review of the email-alert services generated for those sources with no RSS services was more time-consuming. Lessons-for-Guideline-Developers-Adaptors-Implementers-and/orUsers The management of RSS-feeds is considered as a more effective tool to check and follow all the new references published during the guideline development process, being useful also after the CPG publication. Nevertheless, not all databases allowed the creation of RSS feeds, an issue that should be considered by their managers, and in all cases, the updating process has to be supported by professionals.

\section{P198 ADHERENCE TO INFERTILITY GUIDELINES WITH REGARD TO TREATMENT POLICY}

${ }^{1} \mathrm{~F}$ Kersten, ${ }^{1} \mathrm{~W}$ Nelen, ${ }^{2} \mathrm{M}$ Goddijn, ${ }^{1} \mathrm{D}$ Braat, ${ }^{2} \mathrm{~B}$ Mol, R Hermens. ${ }^{1}$ Department of Obstetrics and Gynaecology, Radboud University Nijmegen Medical Centre, Nijmegen, The Netherlands; 'Centre for Reproductive Medicine, Academic Medical Centre, Amsterdam, The Netherlands; ${ }^{3}$ IQ Healthcare, Radboud University Nijmegen Medical Centre, Nijmegen, The Netherlands

\section{0:1136/bmjqs-2013-002293.202}

Background Nowadays, prognostic models are increasingly being used in reproductive medicine. One of these models calculates the chances of conception for couples with unexplained infertility and subsequently indicates the right treatment policy. The Dutch national network guideline on infertility recommends the use of this prognostic model to prevent unnecessary treatment. This strategy is supported by international guidelines on the management of infertility (ESHRE and NICE).

Objectives The aim of this study is to evaluate guideline adherence regarding treatment policy for couples with unexplained infertility in 25 Dutch clinics.
Methods In this retrospective study, we assessed guideline adherence with regard to 1) the use of the prognostic model and 2) the right treatment decision. Data were collected from medical records.

Results In this abstract we present the preliminary data of ten clinics. At the GIN conference we will have the results of all 25 participating clinics. So far we included 208 couples with unexplained infertility. In $54 \%$ of the couples the prognostic model was used. In $69 \%$ of the couples the right treatment decision was primarily made. However, ultimately, $41 \%$ of all couples received unnecessary treatment.

Discussion This study shows that adherence to the guideline regarding treatment policy is not optimal. Despite the guideline recommendations, more than one third of couples are exposed to unnecessary treatment and risks.

Implications for Guideline Developers/Users Implementation of the guideline is not optimal. To improve guideline implementation a multifaceted strategy has to be developed and evaluated.

\section{P199 THE CHALLENGES FOR AN ALCOHOL AND DRUG EDUCATION GUIDELINE FOR COMMUNITY HEALTH WORKERS}

$1,2,3 \mathrm{~A}$ Stein, ${ }^{4} \mathrm{~A}$ Fernandes, ${ }^{5} \mathrm{C}$ Giugliani. ${ }^{1}$ Conceicao Hospital, Porto Alegre, Brazil; ${ }^{2}$ Ufcspa, Porto Alegre, Brazil; ${ }^{3}$ Ulbra, Canoas, Brazil; ${ }^{4}$ Ufrgs, Porto Alegre, Brazil; ${ }^{5}$ Ufrgs, Porto Alegre, Brazil

\section{0:1136/bmjqs-2013-002293.203}

Introduction There is a need to train and develop a guideline for Community Health Workers (CHW) on how to tackle alcohol and drugs problem.

Objective To identify the profile and mental health problems of $\mathrm{CHW}$ and how they manage drug problem.

Methodology A descriptive study was performed and data collected from all $\mathrm{CHW}$ who work at Primary Health Care of Passo Fundo (Brazil). A Self Report Questionnaire (SRQ) was collected to screen mental health on $\mathrm{CHW}$ and scores higher than 7 were identified as positive for mental health problem. Their knowledge about drugs was also collected using a standard questionnaire. The Project was approved by the Passo Fundo University IRB. Descriptive statistics were applied.

Results All $78 \mathrm{CHW}$ were women, age $41.1 \pm 9.6$ years old, $57.7 \%$ have completed high school level, $69.2 \%$ were married and $98.7 \%$ were from lower social class. Half of the CHW were sedentaries, 32\% were smokers, 93.6\% did not report alcohol dependency, although 59\% reported to have a member of their family as having drug or an alcohol problem. They reported that alcohol and drug is a major problem in the community. There were $29.5 \%$ of CHW who have SRQ score higher than 7 . The mean grade of the knowledge test was 34\%.

Conclusion There is a need to develop a guideline on alcohol and drugs for CHW. The profile of CHW had shown a high level of psychiatric problems and lack of basic information.

\section{P200 SUICIDE RISK ASSESSMENT ACCORDING TO BEST PRACTICE GUIDELINES: THE DEVELOPMENT OF A CHART AUDIT PERFORMANCE MEASURE}

${ }^{1}$ E Santa Mina, ${ }^{2} \mathrm{C}$ O'Grady, ${ }^{1} \mathrm{E}$ McCay. ${ }^{1}$ Ryerson University, Toronto, Canada; ${ }^{2}$ Centre for Addiction and Mental Health, Toronto, Canada; ${ }^{3}$ Ryerson University, Toronto, Canada

10:1136/bmjqs-2013-002293.204 